\title{
Optimal Approaches for Measuring Tongue-Pressure Functional Reserve
}

\author{
Catriona M. Steele \\ Toronto Rehabilitation Institute, University Health Network, 550 University Avenue, Toronto, ON, Canada M5G 2A2 \\ Correspondence should be addressed to Catriona M. Steele; catriona.steele@uhn.ca
}

Received 1 November 2012; Accepted 14 January 2013

Academic Editor: Paula Bickford

Copyright (c) 2013 Catriona M. Steele. This is an open access article distributed under the Creative Commons Attribution License, which permits unrestricted use, distribution, and reproduction in any medium, provided the original work is properly cited.

Tongue-palate pressure is a parameter of considerable interest in the field of dysphagia. Maximum isometric tongue-palate pressures (MIPs) decline in healthy aging and in dysphagia. Functional reserve (FR) is the difference between MIPs and swallowing pressures. Reduced FR is thought to constitute a risk for developing functional swallowing impairments. We compare different approaches for calculating FR and recommend an optimal approach. Tongue-palate pressure data were collected from 78 healthy adults ( $40<$ age 40; $38>60$ ) during anterior and posterior MIPs, regular (RESS) and effortful (ESS) saliva swallows, and water swallows (4 repetitions per task). Six different measures of reserve were calculated using maximum anterior MIPs or ESS pressures at the top, and mean or maximum RESS or water swallow pressures at the bottom of the range. Correlations with age and MIPs were explored to confirm suitability for measuring FR. The impact of normalization to maximum MIP range was explored. We conclude that an optimal measure of FR involves the comparison of maximum MIP with mean saliva swallowing pressures. This parameter declines with age, but when normalized to an individual's MIP range, the relationship is no longer evident. This suggests that FR does not necessarily decline in healthy aging.

\section{Introduction}

The ability to generate tongue-palate pressure has emerged as a measure of considerable clinical and research interest in the field of dysphagia over the past two decades. Key to this interest is that tongue strength, measured during maximum isometric tongue-palate pressure tasks (MIPs), appears to decline in healthy aging [1-6]. This has been argued to resemble sarcopenia, a degenerative loss of skeletal muscle mass and strength seen in aging in the limb musculature. Reduced tongue strength has also been observed in adults with dysphagia [7-10]. These observations have prompted research into exercise-based methods for tongue strengthening in the hope that greater tongue strength and endurance will lead to improved swallowing function $[9,11,12]$.

Functional reserve is the term coined to describe the difference in pressures generated in maximum isometric pressure (MIP) tasks compared to swallowing tasks. Robbins and colleagues were the first to point out that swallowing pressures appear to be preserved in healthy older adults, even in the presence of reduced MIPs. Reductions in functional reserve, due to reductions in MIPs, were argued to have important clinical implications and to place a person at greater risk of developing functional swallowing impairments, particularly in the case of decompensation $[2,13]$.

Functional reserve describes a tongue-palate pressure range that is bounded at the top by a maximum pressure task and at the bottom by a swallowing task. The swallowing task used to define the bottom of the functional reserve range has varied across studies, leading to some confusion regarding the measure. Furthermore, different instruments have been used across studies, without clear demonstration that measures can be generalized across instruments. We are conducting a program of research exploring the relationship between tongue pressure capacity and swallowing behaviors. We are specifically interested in confirming whether or not limited tongue pressure capacity contributes to constrained variability in swallowing behaviors and whether such constraints are seen as a function of healthy aging. We have recently reported that functional reserve, which we defined as the difference between MIPs and regular effort saliva swallow (RESS) pressures, does not necessarily decline with age 
[14]. Although overall groupwise trends showed significantly larger functional reserve in individuals aged 20-40 compared to those over the age of 60 , we noticed that $18 \%$ of our older participant group had excellent functional reserve above the 75th percentile for pooled functional reserve in our sample. Similarly, $13 \%$ of our younger group had limited functional reserve, below the 25 th $\%$ percentile.

Of particular note is the study of Youmans et al. [5], which involved a functional reserve calculation using bolus swallowing pressures at the bottom of the range, with these pressure measures transformed to a percentage value relative to each participant's MIP range, thereby controlling individual differences in strength. These authors found no significant age-group differences using this transformation, although women were found to use a higher percentage of their MIP range when swallowing boluses compared to men. Youmans and colleagues argued that these results challenged the conventional view that functional reserve declines in healthy aging. Similarly, a previous study by Yeates and colleagues [15] measured a parameter that they called "swallow reserve," comparing effortful saliva swallows to regular effort saliva swallows, and failed to find age-related differences in this measure.

These observations led us to question whether functional reserve is indeed a parameter that changes in healthy aging and to explore which elements need to be included in a robust and psychometrically valid measure of functional tongue pressure reserve. We propose that such a parameter should display three characteristics.

(a) A measure of functional reserve should be sensitive to, and positively correlated with, changes in maximum strength measures (MIPs).

(b) A measure of functional reserve should be sensitive to changes that occur across the age span, but in order for these to support a hypothesis that functional reserve declines with age, the trend should be visible in a parameter where swallowing pressures are expressed as a percentage of a person's maximum tongue strength, in order to control for individual variations in strength.

(c) A measure of functional reserve should be one that can be collected easily, without posing a risk of aspiration for the patient.

In this paper, we explore 6 different candidate measures of functional reserve to determine an optimum method for capturing functional reserve in future research and clinical situations.

\section{Materials and Methods}

Tongue pressures at the anterior, medial, and posterior palate were collected from 78 healthy consenting adults in two sexbalanced age groups. The younger participant group ranged in age from 18 to 39 years, with a mean age of 27 years. The older participant group ranged in age from 60 to 87, with a mean age of 70 years. The protocol was approved by the local institutional research ethics board. Exclusion criteria

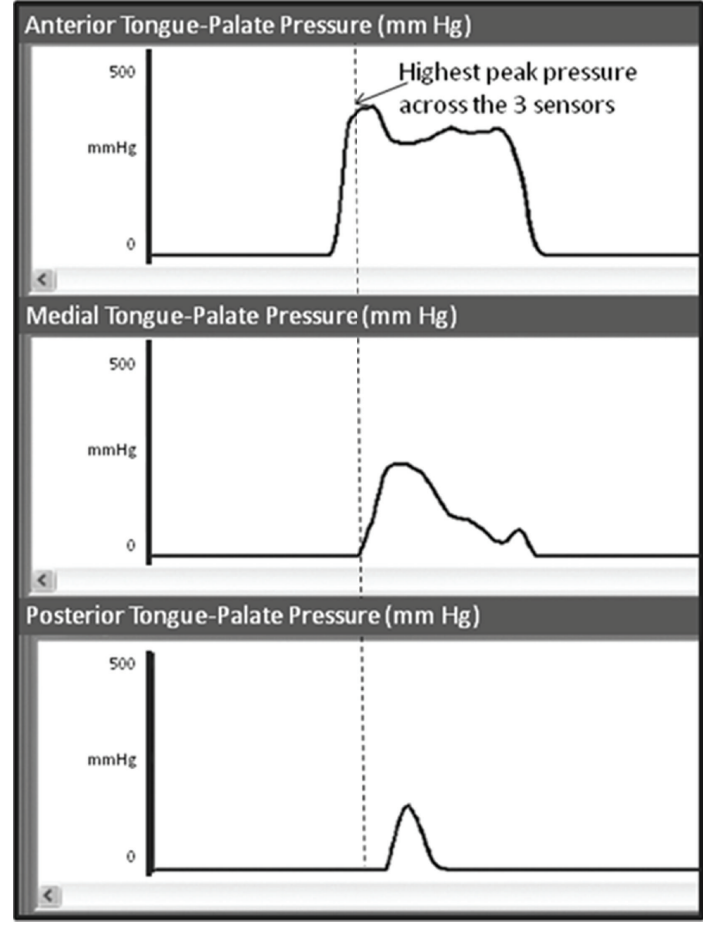

FIGURE 1: Illustration of tongue-palate pressure waveforms on the KayPentax Digital Swallow Workstation. The highest peak pressure (in $\mathrm{mm} \mathrm{Hg}$ ) across the anterior, medial, and posterior sensors was used as the value of peak pressure for each task.

included participant-reported history of type I diabetes, chronic sinusitis, taste disturbance, or any swallowing, motor speech, gastroesophageal, or neurological difficulties. During intake, a brief oral mechanism examination and a water swallow screening were performed by a licensed speechlanguage pathologist to confirm eligibility to participate.

2.1. Data Collection. We used the 3-bulb tongue array of the KayPentax Swallowing Signals Lab to collect tongue pressures, with the bulbs spaced $8 \mathrm{~mm}$ apart and adhered to the palate in midline using Stomahesive (Convatec, St-Laurent, Quebec, Canada). The anterior bulb was positioned on the alveolar ridge, immediately behind the upper incisors. Pressure signals were sampled at $250 \mathrm{~Hz}$ with the range calibrated to record an upper amplitude limit of $750 \mathrm{mmHg}$. Five different pressure tasks were included, with each performed in a block of 4 task repetitions. The protocol commenced with the maximum anterior isometric pressure task (AMAX) and then proceeded to one of four randomly assigned sequences for the collection of regular effort saliva swallows (RESS), effortful saliva swallows (ESS), maximum posterior isometric pressures (PMAX), and discrete water swallows (i.e., single sips of $\sim 8-10 \mathrm{~mL}$ taken from a cup). The PMAX data have been reported elsewhere and are not used for the calculations reported in this paper.

2.2. Data Processing. Anterior, medial, and posterior palate pressure waveforms were displayed on a computer monitor. The onset, peak, and offset of each pressure event were 
TABLE 1: Pearson's correlations between different measures of functional reserve: maximum isometric pressure and age.

\begin{tabular}{|c|c|c|c|c|c|}
\hline \multirow{2}{*}{ Functional reserve measure } & \multirow{2}{*}{$\begin{array}{c}\text { Unit of } \\
\text { measurement }\end{array}$} & \multicolumn{2}{|c|}{ Maximum isometric pressure (MIP) } & \multicolumn{2}{|c|}{ Age } \\
\hline & & $\begin{array}{l}\text { Pearson's } \\
\text { correlation }\end{array}$ & $\begin{array}{l}\text { Significance } \\
\text { (2-tailed) }\end{array}$ & $\begin{array}{l}\text { Pearson's } \\
\text { correlation }\end{array}$ & $\begin{array}{l}\text { Significance } \\
\text { (2-tailed) }\end{array}$ \\
\hline \multirow{2}{*}{ MIP minus maximum water swallow pressures } & $\mathrm{mm} \mathrm{Hg}$ & .814 & .000 & -.304 & .007 \\
\hline & $\%$ MIP & .314 & .005 & -.104 & .368 \\
\hline \multirow{2}{*}{ MIP minus mean water swallow pressures } & $\mathrm{mm} \mathrm{Hg}$ & .910 & .000 & -.359 & .001 \\
\hline & $\%$ MIP & .320 & .005 & -.107 & .354 \\
\hline \multirow{2}{*}{$\begin{array}{l}\text { MIP minus maximum regular effort saliva swallow } \\
\text { pressures }\end{array}$} & $\mathrm{mm} \mathrm{Hg}$ & .796 & .000 & -.250 & .031 \\
\hline & $\%$ MIP & .453 & .000 & -.042 & .716 \\
\hline \multirow{2}{*}{ MIP minus mean regular effort saliva swallow pressures } & $\mathrm{mm} \mathrm{Hg}$ & .899 & .000 & -.312 & .006 \\
\hline & $\%$ MIP & .493 & .000 & -.078 & .500 \\
\hline $\begin{array}{l}\text { Maximum effortful minus maximum regular effort } \\
\text { saliva swallow pressures }\end{array}$ & $\mathrm{mm} \mathrm{Hg}$ & .285 & .013 & $\mathrm{n} / \mathrm{a}$ & $\mathrm{n} / \mathrm{a}$ \\
\hline $\begin{array}{l}\text { Maximum effortful minus mean regular effort saliva } \\
\text { swallow pressures }\end{array}$ & $\mathrm{mm} \mathrm{Hg}$ & .325 & .004 & $\mathrm{n} / \mathrm{a}$ & $\mathrm{n} / \mathrm{a}$ \\
\hline
\end{tabular}

Correlations with $R>0.4$ are shown in bold.

indexed by a trained research assistant and pressure amplitudes at each of these timepoints were recorded. Pressure amplitude (in $\mathrm{mm} \mathrm{Hg}$ ) was calculated as the amplitude difference (in $\mathrm{mm} \mathrm{Hg}$ ) between the highest peak pressure amplitude and the lowest baseline pressure amplitude (usually zero $\mathrm{mm} \mathrm{Hg}$ ) seen across all three pressure waveforms (anterior, medial, and posterior) for a given task repetition. Figure 1 provides an illustration of this parameter.

2.3. Analysis. Six different equations were calculated as possible measures of functional reserve as follows.

(a) Maximum peak pressure on the AMAX task minus maximum peak pressure on the water swallowing task.

(b) Maximum peak pressure on the AMAX task minus mean peak pressure on the water swallowing task.

(c) Maximum peak pressure on the AMAX task minus maximum peak pressure on the RESS task.

(d) Maximum peak pressure on the AMAX task minus mean peak pressure on the RESS task.

(e) Maximum peak pressure on the ESS task minus maximum peak pressure on the RESS task.

(f) Maximum peak pressure on the ESS task minus mean peak pressure on the RESS task.

Scatter plots were prepared in IBM SPSS 19.0, plotting each measure of functional reserve, first as a function of maximum isometric pressure (maximum peak pressure on the AMAX task) and second as a function of age. An a priori criterion for correlations of interest was established as a Pearson's correlation of $R=0.4$. Finally, RESS and water swallow pressure values were transformed to a percentage value of each participant's maximum isometric pressures, and scatter plots and correlations were again explored using these transformed data.

\section{Results and Discussion}

Table 1 provides descriptive statistics for the observed Pearson's correlations between each candidate measure of functional reserve: (a) maximum isometric pressure and (b) age.

As shown in Figure 2(a), when the traditional approach for measuring functional reserve is used (MIP minus water swallowing pressures), there are strong correlations with MIP, regardless of whether mean $\left(R^{2}=0.85\right)$ or maximum $\left(R^{2}=0.70\right)$ water swallowing pressure values are used at the bottom of the functional reserve range. Similarly, as shown in Figure 2(b), strong correlations are also seen when either mean $\left(R^{2}=0.81\right)$ or maximum $\left(R^{2}=0.64\right)$ saliva swallow pressures are used at the bottom of the functional reserve range. By contrast, as shown in Figure 2(c), measures of swallow reserve, in which effortful saliva swallows are used at the top of the functional reserve range, do not correlate strongly with MIP when either mean $\left(R^{2}=0.10\right)$ or maximum $\left(R^{2}=0.07\right)$ saliva swallows are used at the bottom of the range. This suggests that swallow reserve, as proposed by Yeates and colleagues [15], is not sensitive to differences in tongue pressure capacity and is therefore not a good measure of functional reserve.

With respect to the relationship between functional reserve measures and age, Figure 3(a) shows weak but statistically significant negative correlations with age for functional reserve measures comparing MIP to mean $\left(R=-0.31, R^{2}=\right.$ $0.13)$ or maximum $\left(R=-0.25, R^{2}=0.10\right)$ water swallowing pressures. Similarly, as shown in Figure 3(b), weak but statistically significant correlations with age are seen when either mean $\left(R=-0.36, R^{2}=0.10\right)$ or maximum $(R=-0.30$, $\left.R^{2}=0.07\right)$ saliva swallow pressures are used at the bottom of the functional reserve range. Age correlations with swallow reserve were not explored given the earlier result showing a lack of sensitivity to changes in strength.

Finally, we explored what happened to the previously observed correlations when we transformed our measures of 


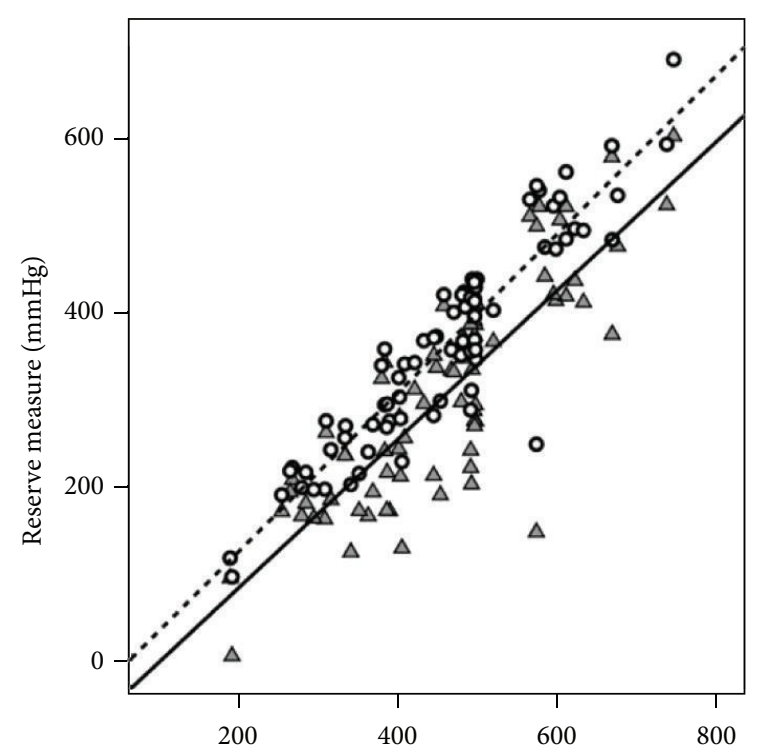

Tongue strength (maximum isometric pressure $(\mathrm{mmHg})$ )
MIP minus mean water swallow pressures: $R^{2}=0.85$

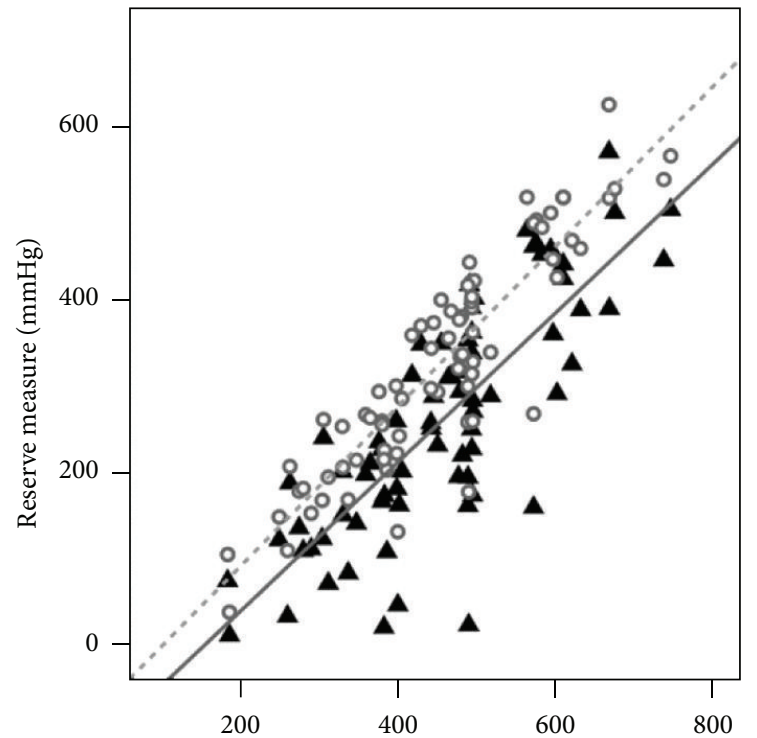

Tongue strength (maximum isometric pressure $(\mathrm{mmHg})$ )

O MIP minus mean regular effort saliva swallow pressures: $R^{2}=0.81---$
A MIP minus maximum regular effort saliva swallow pressures: $R^{2}=0.64$

(a)

(b)

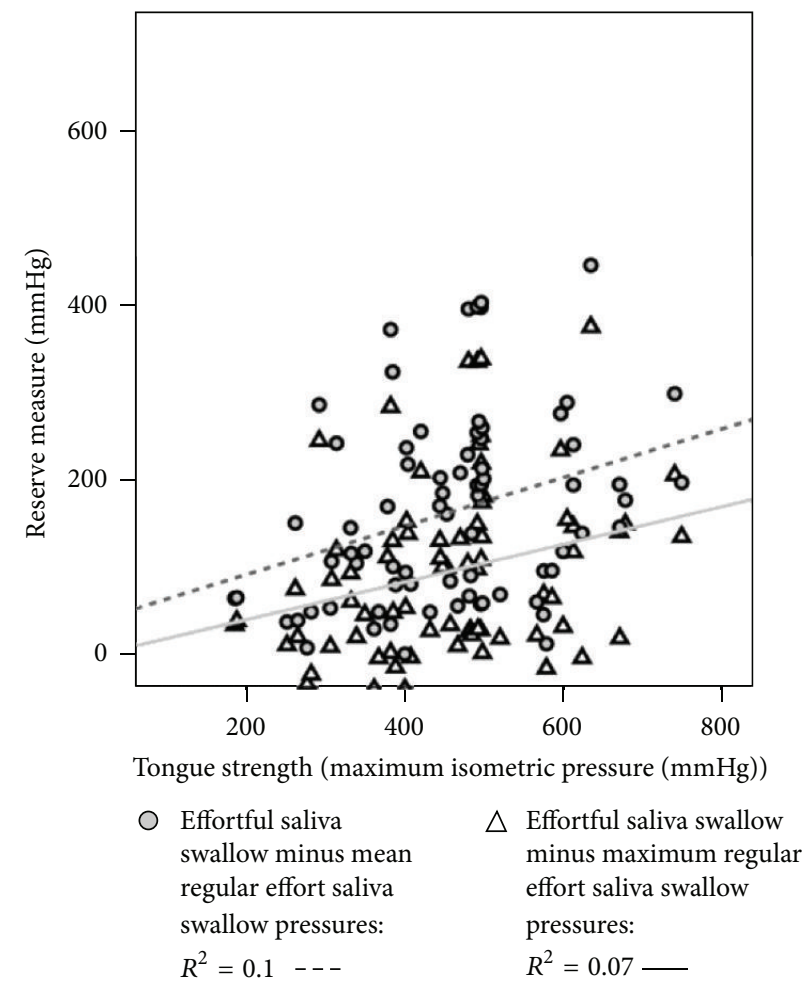

(c)

FIgURE 2: (a) Scatter plot showing the relationship between functional reserve measures comparing maximum isometric pressure (MIP) to water swallowing pressures and tongue strength, as measured by MIP. (b) Scatter plot showing the relationship between functional reserve measures comparing maximum isometric pressure (MIP) to regular effort saliva swallowing pressures and tongue strength, as measured by MIP. (c) Scatter plot showing the relationship between functional reserve measures comparing maximum effortful saliva swallowing pressures to regular effort saliva swallowing pressures and tongue strength, as measured by MIP. 


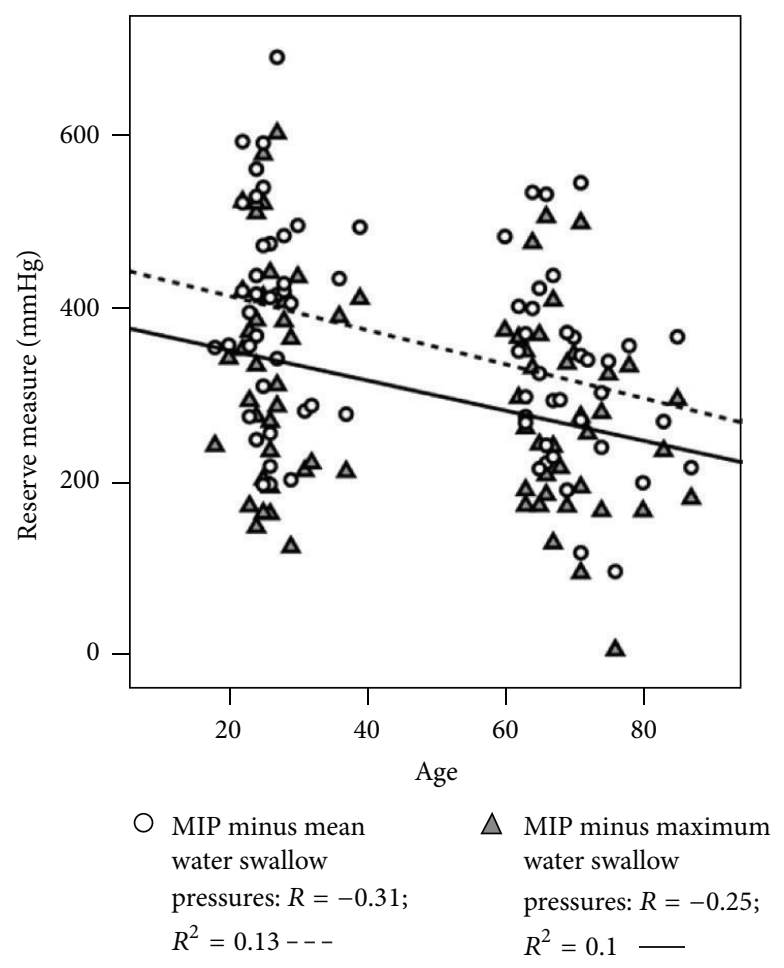

(a)

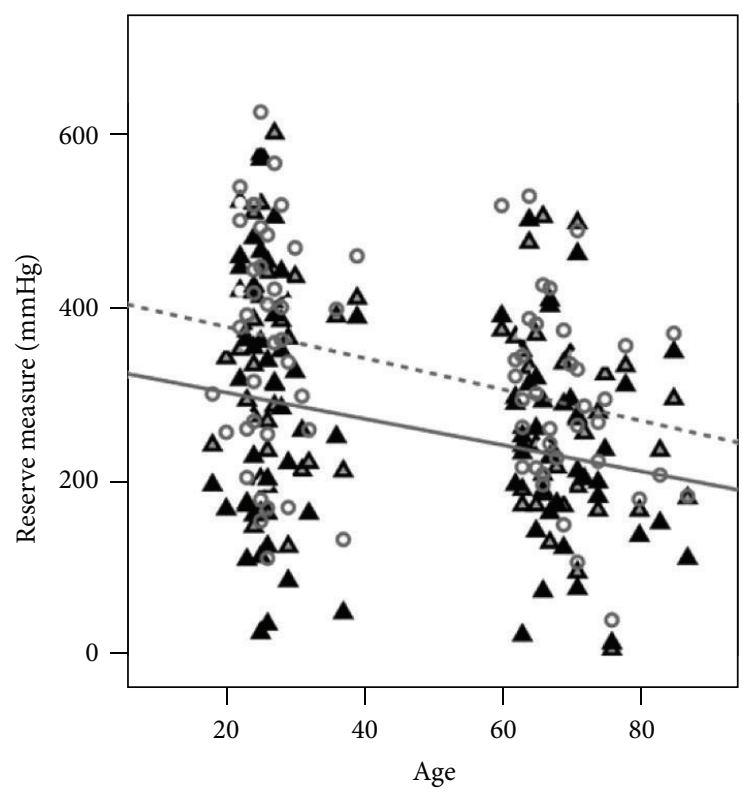

$\begin{array}{ll}\begin{array}{l}\text { MIP minus mean } \\ \text { regular effort saliva }\end{array} & \boldsymbol{\Delta} \begin{array}{l}\text { MIP minus maximum } \\ \text { regular effort saliva }\end{array} \\ \text { swallow pressures: } & \text { swallow pressures: } \\ R=-0.36 ; R^{2}=0.1^{---} & R=-0.3 ; R^{2}=0.07\end{array}$

(b)

FIGURE 3: (a) Scatter plot showing the relationship between functional reserve measures comparing maximum isometric pressure (MIP) to water swallowing pressures and age. (b) Scatter plot showing the relationship between functional reserve measures comparing maximum isometric pressure (MIP) to regular effort saliva swallowing pressures and age.

swallowing pressure into values normalized to a percentage of each participant's maximum strength measure. As shown in Figure 4(a), strong correlations with MIP are retained with this transformation when saliva swallows are used at the bottom of the functional reserve range. With water swallows, the correlations fall just below the a priori criterion of $R=$ 0.4 , but remain statistically significant (Figure $4(\mathrm{~b})$ ). With respect to correlations with age, if a correlation age remains significant after this transformation, we would be able to conclude that functional reserve declines as a function of age, rather than being primarily driven by a person's strength. This, however, was not the case. Figures 5(a) and 5(b) show that the previously observed weak correlations with age are negated by the transformation. No statistically significant correlations with age are seen, regardless of the equation used to calculate functional reserve. This result confirms the previous result reported by Youmans and colleagues [5], suggesting that the appearance of an age-related decline in functional reserve may be an artifact attributable to variability in MIP measures across participants.

\section{Conclusions}

We conclude that optimally robust measures of functional reserve are those comparing maximum tongue strength (MIPs) to mean water or saliva swallowing pressures. Of these two options, saliva swallows involve less risk of aspiration during data collection. The results of the analysis in this study suggest that it would be reasonably straight forward for clinicians to routinely incorporate the measurement of tongue pressure functional reserve into swallowing assessment, using a short series of MIPs and saliva swallows.

We recommend that functional reserve measures should use a data transformation to account for individual differences in maximum tongue strength measures, by expressing swallowing pressures as a percent of a person's MIP. Using this transformation, functional reserve measures comparing MIP to either mean or maximum saliva swallow pressure retain statistically significant strong correlations with strength. However, this transformation has the effect of negating apparent age-related differences in functional reserve.

When normalized to control individual variations in strength, our data concur with those of Youmans and colleagues [5] in showing that functional reserve does not decline as a function of healthy aging. This finding strongly suggests that older adults should not be assumed to have tongue weakness and reduced functional reserve simply as a function of age. Indeed, our data suggest that the distribution of reduced functional reserve is not age dependent and that individuals with limited functional reserve may be found in the younger population. Certainly, prior studies point to limited functional reserve being a characteristic of dysphagia. The consequences of functional reserve for swallowing function and the ability to benefit from commonly used 


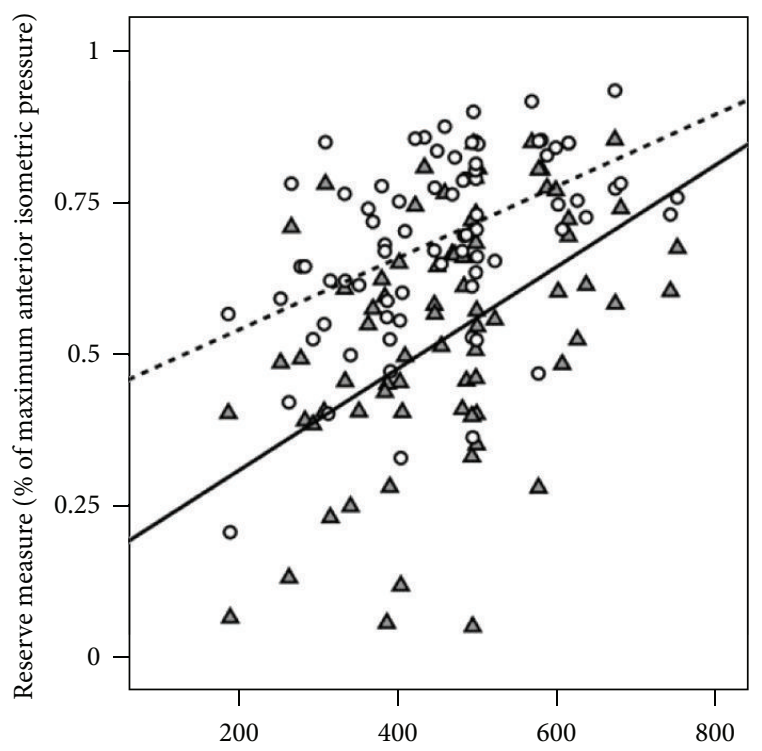

Tongue strength (maximum isometric pressure $(\mathrm{mmHg})$ )

$\begin{array}{ll}\text { MIP minus mean } & \Delta \text { MIP minus maximum } \\ \text { water swallow } & \text { water swallow pressures: } \\ \text { pressures: } R^{2}=0.103-\ldots & R^{2}=0.099\end{array}$

(a)

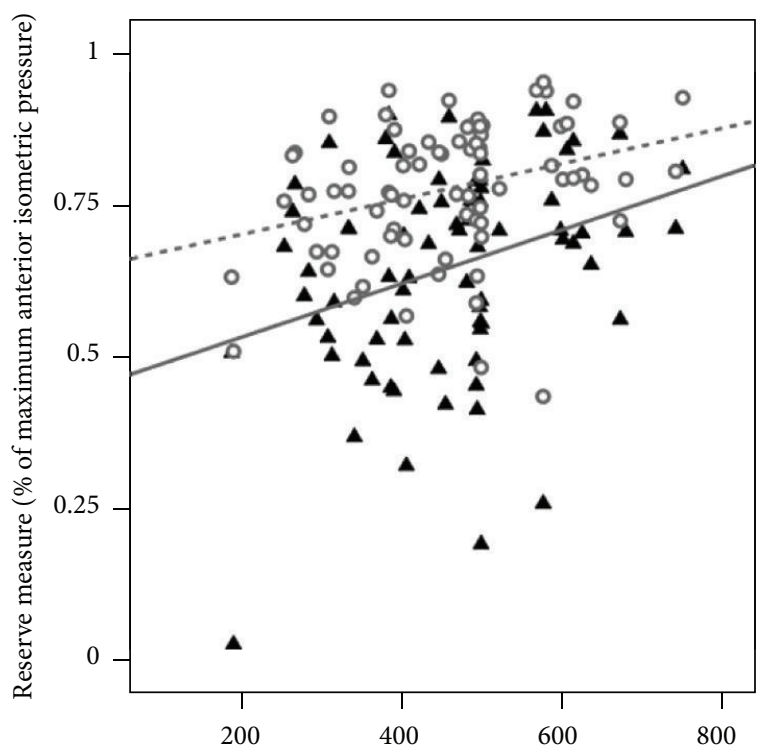

Tongue strength (maximum isometric pressure $(\mathrm{mmHg})$ )
O MIP minus mean regular effort saliva swallow pressures: $R^{2}=0.24$
\ MIP minus maximum regular effort saliva swallow pressures: $R^{2}=0.21-$ (b)

FIGURE 4: (a) Scatter plot showing the relationship between functional reserve measures comparing maximum isometric pressure (MIP) to water swallowing pressures and tongue strength, as measured by MIP, with swallow pressures expressed as a percentage of MIP. (b) Scatter plot showing the relationship between functional reserve measures comparing maximum isometric pressure (MIP) to regular effort saliva swallowing pressures and tongue strength, as measured by MIP, with swallow pressures expressed as a percentage of MIP.

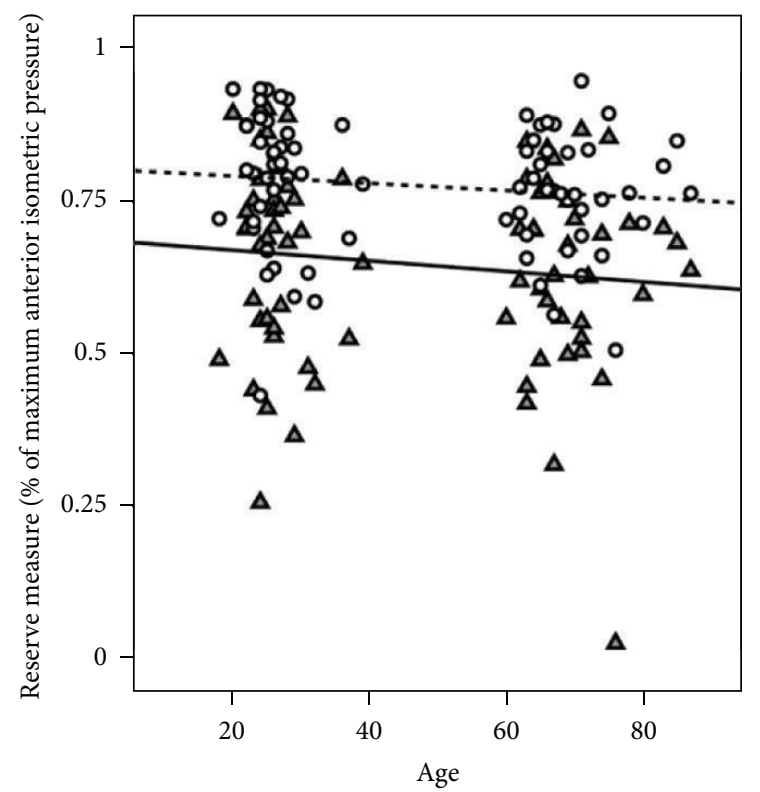

$$
\begin{aligned}
& \text { MIP minus mean water } \triangle \text { MIP minus maximum } \\
& \text { swallow pressures: } \\
& R=-0.08 \text { (nonsignificant); } \\
& R^{2}=0.02 \\
& R^{2}=0.01
\end{aligned}
$$

(a)

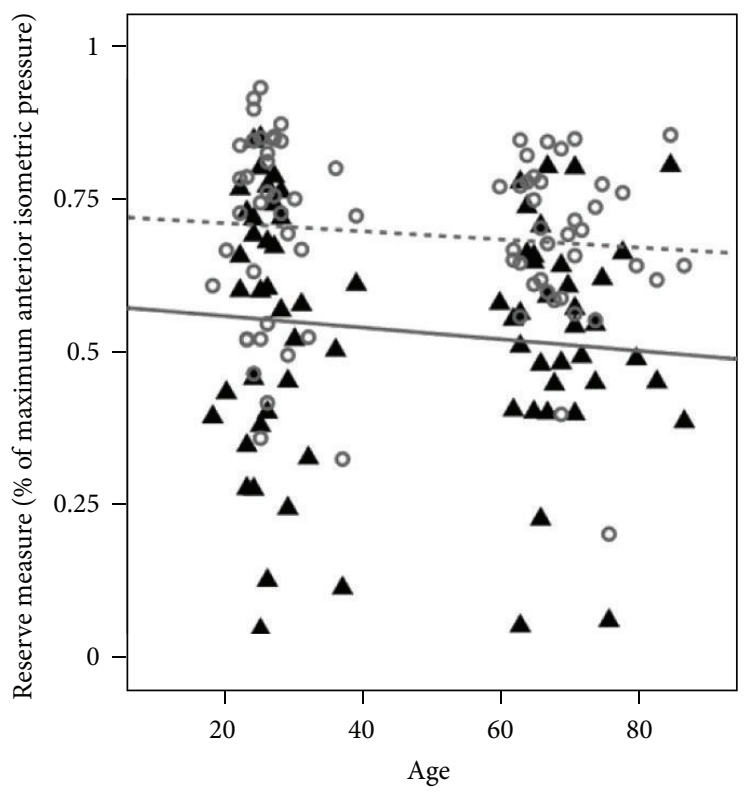

MIP minus mean water swallow pressures: $R=-0.11$ (nonsignificant); $R^{2}=0.01$
A MIP minus maximum water swallow pressures: $R=-0.1$ (nonsignificant); $R^{2}=0.01$ (b)

FIGURE 5: (a) Scatter plot showing the relationship between functional reserve measures comparing maximum isometric pressure (MIP) to water swallowing pressures and age, with swallow pressures expressed as a percentage of MIP. (b) Scatter plot showing the relationship between functional reserve measures comparing maximum isometric pressure (MIP) to regular effort saliva swallowing pressures and age, with swallow pressures expressed as a percentage of MIP. 
interventions such as texture modification require further elucidation in future studies. Similarly, the benefits of tongue pressure resistance training for building functional reserve and restoring more normal swallowing function are still to be clearly demonstrated.

\section{Acknowledgments}

This work was supported by the National Institutes of Health (Grant no. 5R01DC011020 to C. M. Steele); and from the Toronto Rehabilitation Institute, which receives funding under the Provincial Rehabilitation Research Program from the Ontario Ministry of Health and Long-Term Care (MOHLTC). The views expressed do not necessarily reflect those of the ministry. The author would like to thank Drs. Mark Bayley, Cathy Pelletier, and Julie Cichero for their contributions to the study design and interpretation. Assistance from Rebecca Cliffe Polacco, Sarah Hori, Sonja Molfenter, Melanie Peladeau-Pigeon, and Clemence Tsang during data collection and processing is gratefully acknowledged.

\section{Conflict of Interests}

There is no conflict of interests to disclose.

\section{References}

[1] H. C. Crow and J. A. Ship, "Tongue strength and endurance in different aged individuals," Journals of Gerontology. Series A, vol. 51, no. 5, pp. M247-M250, 1996.

[2] M. A. Nicosia, J. A. Hind, E. B. Roecker et al., "Age effects on the temporal evolution of isometric and swallowing pressure," Journals of Gerontology. Series A, vol. 55, no. 11, pp. M634-M640, 2000.

[3] S. R. Youmans and J. A. G. Stierwalt, "Measures of tongue function related to normal swallowing," Dysphagia, vol. 21, no. 2, pp. 102-111, 2006.

[4] Y. Utanohara, R. Hayashi, M. Yoshikawa, M. Yoshida, K. Tsuga, and Y. Akagawa, "Standard values of maximum tongue pressure taken using newly developed disposable tongue pressure measurement device," Dysphagia, vol. 23, no. 3, pp. 286-290, 2008.

[5] S. R. Youmans, G. L. Youmans, and J. A. G. Stierwalt, "Differences in tongue strength across age and gender: is there a diminished strength reserve?" Dysphagia, vol. 24, no. 1, pp. 57-65, 2009.

[6] K. Tamine, T. Ono, K. Hori, J. Kondoh, S. Hamanaka, and Y. Maeda, "Age-related changes in tongue pressure during swallowing," Journal of Dental Research, vol. 89, no. 10, pp. 1097-1101, 2010.

[7] R. White, S. M. Cotton, J. Hind, J. Robbins, and A. Perry, "A comparison of the reliability and stability of oro-lingual swallowing pressures in patients with head and neck cancer and healthy adults," Dysphagia, vol. 24, no. 2, pp. 137-144, 2009.

[8] S. Ball, O. Idel, S. M. Cotton, and A. Perry, "Comparison of two methods for measuring tongue pressure during swallowing in people with head and neck cancer," Dysphagia, vol. 21, no. 1, pp. 28-37, 2006.

[9] J. Robbins, S. A. Kays, R. E. Gangnon et al., "The effects of lingual exercise in stroke patients with dysphagia," Archives of Physical Medicine and Rehabilitation, vol. 88, no. 2, pp. 150-158, 2007.
[10] N. Hirota, K. Konaka, T. Ono et al., "Reduced tongue pressure against the hard palate on the paralyzed side during swallowing predicts Dysphagia in patients with acute stroke," Stroke, vol. 41, no. 12, pp. 2982-2984, 2010.

[11] J. Robbins, R. E. Gangnon, S. M. Theis, S. A. Kays, A. L. Hewitt, and J. A. Hind, "The effects of lingual exercise on swallowing in older adults," Journal of the American Geriatrics Society, vol. 53, no. 9, pp. 1483-1489, 2005.

[12] C. L. Lazarus, J. A. Logemann, C. F. Huang, and A. W. Rademaker, "Effects of two types of tongue strengthening exercises in young normals," Folia Phoniatrica et Logopaedica, vol. 55, no. 4, pp. 199-205, 2003.

[13] J. Robbins, R. Levine, J. Wood, E. B. Roecker, and E. Luschei, "Age effects on lingual pressure generation as a risk factor for dysphagia," Journals of Gerontology. Series A, vol. 50, no. 5, pp. M257-M262, 1995.

[14] T. Fei, R. Cliffe Polacco, S. M. Molfenter, M. Peladeau-Pigeon, C. Tsang, and C. M. Steele, "Age-related differences in tonguepressure release-slope for saliva and water swallows," in Proceedings of the 20th Annual Meeting of the Dysphagia Research Society, Springer, Toronto, Canada, 2012.

[15] E. M. Yeates, C. M. Steele, and C. A. Pelletier, "Tongue pressure and submental surface electromyography measures during noneffortful and effortful saliva swallows in healthy women," American Journal of Speech-Language Pathology, vol. 19, no. 3, pp. 274-281, 2010. 


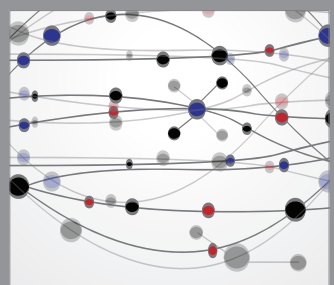

The Scientific World Journal
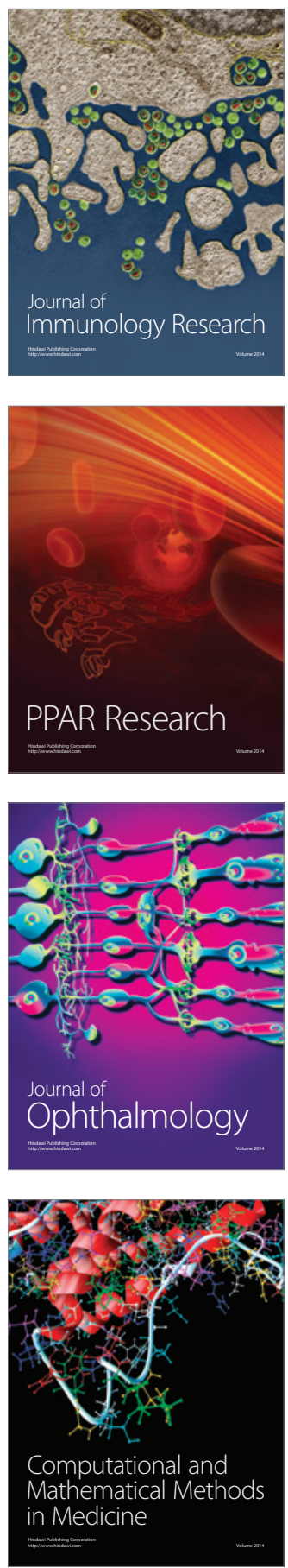

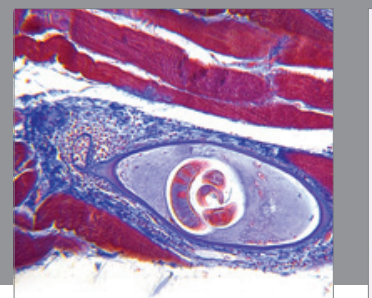

Gastroenterology

Research and Practice
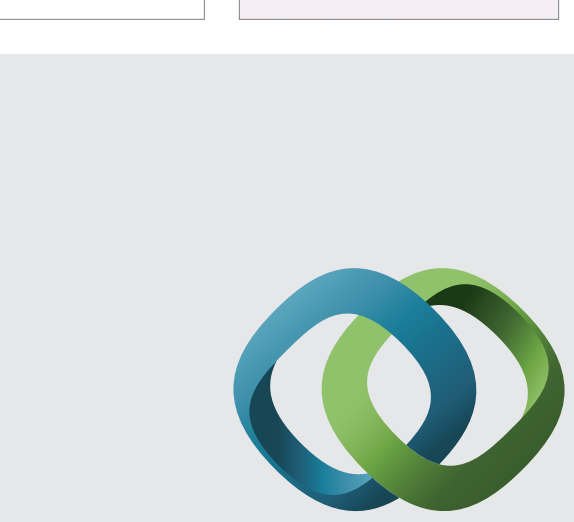

\section{Hindawi}

Submit your manuscripts at

http://www.hindawi.com
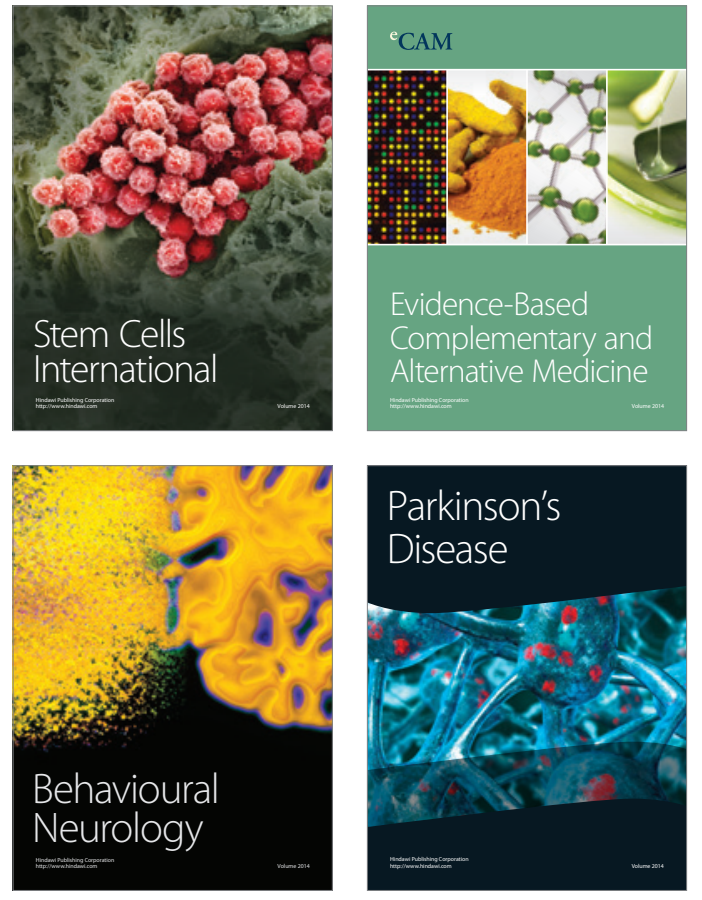
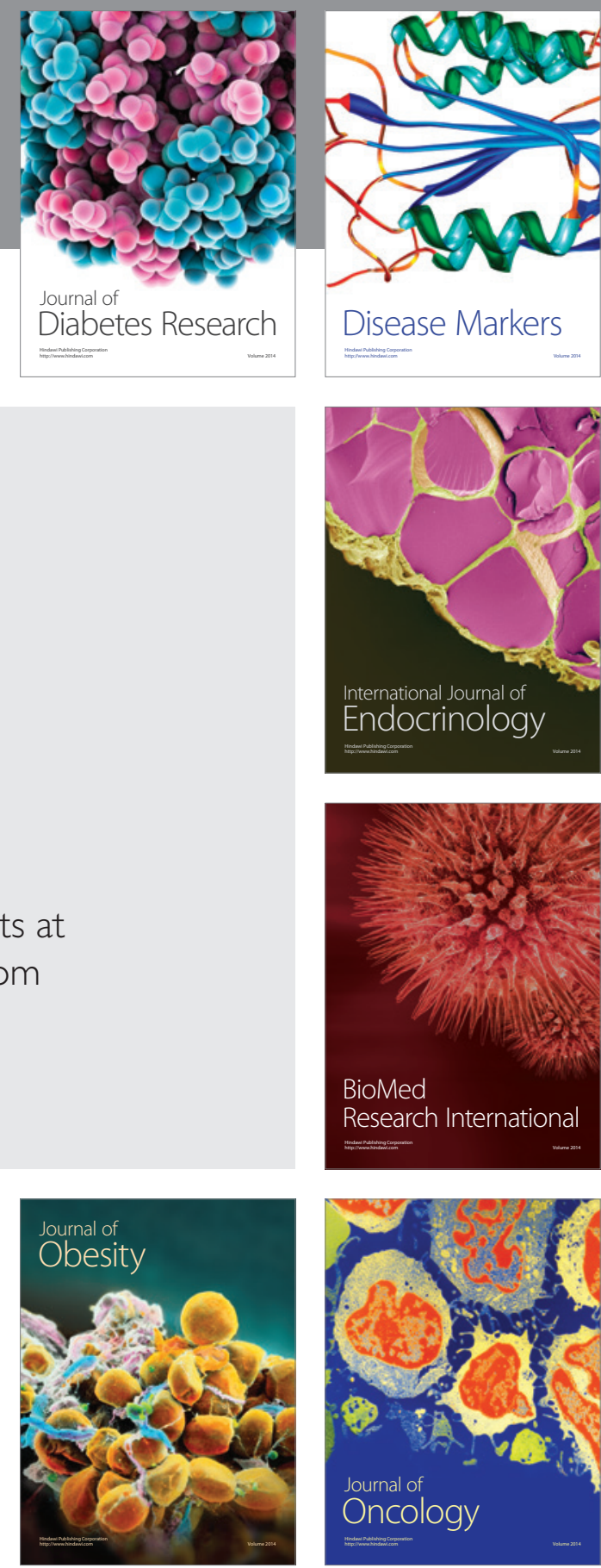

Disease Markers
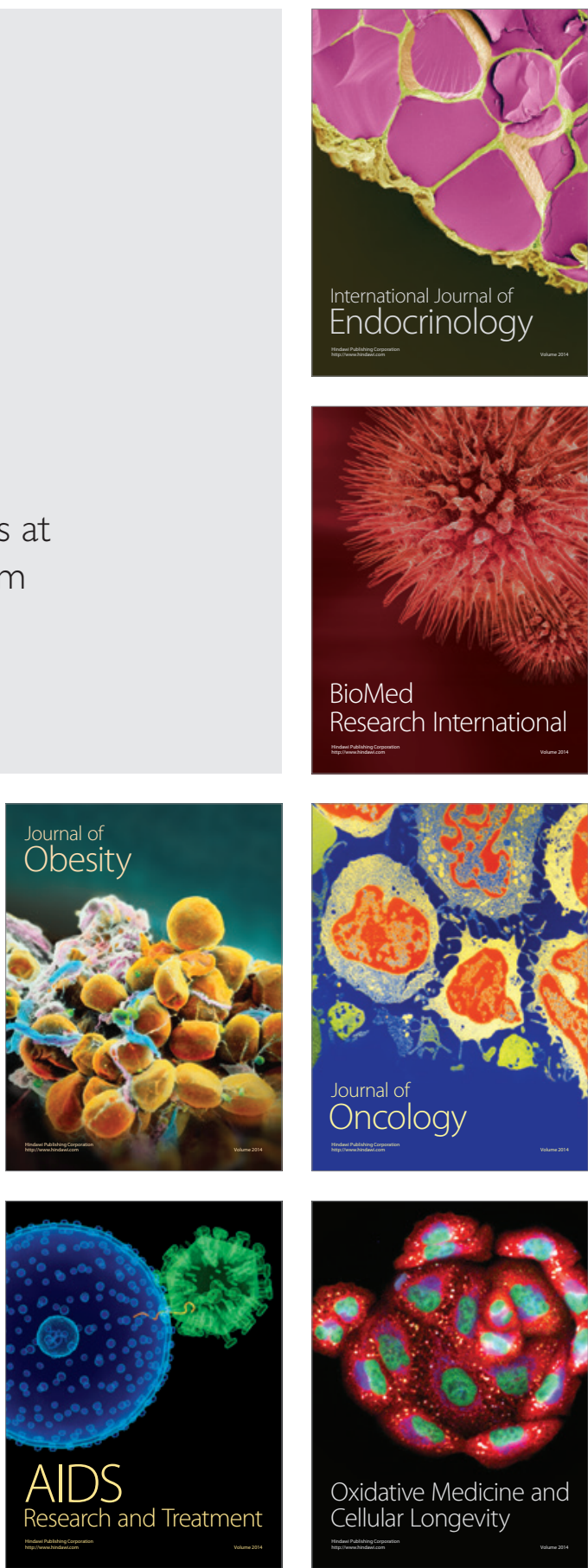